\title{
Parametric Study of Spot Welding between Li-ion Battery Cells and Sheet Metal Connectors
}

\author{
Manop Masomtob ${ }^{1,3,4, a, *}$, Rawitat Sukondhasingha ${ }^{1}$, Jan Becker ${ }^{1,3}$, and Dirk Uwe Sauer ${ }^{1,2,3, b}$ \\ 1 Electrochemical Energy Conversion and Storage Systems Group, Institute for Power Electronics and \\ Electrical Drives (ISEA), RWTH Aachen University, Aachen, 52066, Germany \\ 2 Institute for Power Generation and Storage Systems (PGS), E.ON ERC, RWTH Aachen University, \\ Aachen, 52074, Germany \\ 3 Juelich Aachen Research Alliance, JARA-Energy, Germany \\ 4 Materials for Energy Research Unit, National Metal and Materials Technology Center (MTEC), National \\ Science and Technology Development Agency (NSTDA), Pathum Thani 12120, Thailand \\ E-mail: amanopm@mtec.or.th (Corresponding author), bbatteries@isea.rwth-aachen.de
}

\begin{abstract}
This work was designed to study the effects of influencing parameters in series/parallel gap spot welding process and determine the optimized parameters setting for spot welding between $18650 \mathrm{Li}$-ion battery cells and sheet metal connectors. The welding parameters that were studied in this work include electrode tip geometry, connecting strip material and design, maximum supply voltage, welding time welding force and distance between two electrodes. The effects of these parameters were investigated via simulations and experimentations. A sample battery cell was disassembled and measured to create the 3D models. Simulations were carried out software to observe the deformation, electric current distribution as well as temperature distribution occurred within the models. For experiments done in this work, the actual spot welding was conducted using the Miyachi FD120 series spot welding machine. The trial welding was first performed between two different thicknesses of Hilumin ${ }^{\circledR}$ connecting strips themselves. Then, after having the results of studied parameters from both simulations and experiments compared and analyzed, the actual spot welding was conducted between Hilumin ${ }^{\circledR}$ connecting strips and both of positive and negative terminals of SAMSUNG INR18650-15L Li-ion battery cells. The electric resistance and mechanical strength of the welded contact were measured. Finally, the suistriple welding parameter setting ranges were obtained as a result, which can be applied to create battery packs either from the similar or other different models of 18650 Li-ion cells via series spot welding processes.
\end{abstract}

Keywords: Spot welding, Li-ion battery cell, hilumin sheet metal connector (nickel-plated steel).

ENGINEERING JOURNAL Volume 21 Issue 7

Received 1 September 2017

Accepted 18 October 2017

Published 29 December 2017

Online at http://www.engj.org/

DOI:10.4186/ej.2017.21.7.457 


\section{Introduction}

Nowadays, Li-ion battery cells have been used in many applications such as pure electric/hybrid vehicles, electronic mobile devices and etc. because of high energy density. However, one single battery cell cannot be used in a battery pack because its voltage and current density are not enough for operation. Therefore, many battery cells must be assembled and connected in serial/parallel for battery packs. Consequently, many electrical connectors are required as electrical bridges between battery cells. For most 18650 Li-ion battery cells, either spot or laser welding technique can be used to weld a sheet metal connector with a battery cell. In general, the spot welding technique is widely used more than the laser welding technique because its infrastructure cost is lower [1]. However, the quality of the spot welding technique is lower than which of the laser welding technique because there are many parameters which are necessary to be controlled such as welding force from electrode welder, electric voltage, electric current, welding time and electrode geometry as well as electrode material. Therefore, this paper will study several crucial parameters in order to obtain good electrical contact and mechanical strength of contact for the spot welding technique.

\section{Parameter Study}

In this work, eight parameters which are electrode tip geometry, distance between two welding electrodes, connecting strip design, connecting strip material, welding force, maximum voltage and welding time, were studied by using simulations and experiments. The simulation was used to investigate some behaviors that cannot be analyzed using data from the experiment such as the current direction, the temperature distribution on spot welding and the deformation. The experiment was performed in parallel as to prove the strength and the quality of welding contacts. After the parameters have been studied, suitable parameters are also introduced in this paper in order to be guide line for application to create battery packs either from the similar or other different models of 18650 Li-ion cells.

\section{Materials}

There are three main materials in each component as shown in Fig. 1.

\subsection{Electrode Welder}

The electrode welder is made of Copper, Chromium, and Zirconium $(\mathrm{CuCrZr})$ is a suistriple welding electrode material used for spot welding the work-pieces made of stainless steel and Nickel alloys [2].

\subsection{Battery Case}

The battery case is the housing case of Samsung INR18650-15L battery cell; therefore, the exact type could not be identified. Based on the cost-effective and a high availability in the market of the Nickel-coated steel strips, which are commonly called under the brand name Hilumin ${ }^{\circledR}$ developed by the Corus Special strip. Hilumin ${ }^{\circledR}$ is an electrolytically Nickel-plated, diffusion-annealed cold rolled steel strip specifically for battery applications where low contact resistance and high corrosion resistance is required. It is widely used as battery cans (Alkaline, NiMH, NiCd, LiIon, and ZincAir), battery covers, and connecting strips [3].

\subsection{Connecting Strip/Tab}

The connecting strip/tab which is made of Hilumin ${ }^{\circledR}$ or pure nickel can be found in markets. Generally, Hilumin ${ }^{\circledR}$ is cheaper than nickel. In some countries, their prices are not different. In fact, pure nickel strip is better than Hilumin ${ }^{\circledR}$ in term of electrical conductivity, thermal conductivity and oxidation rate. 

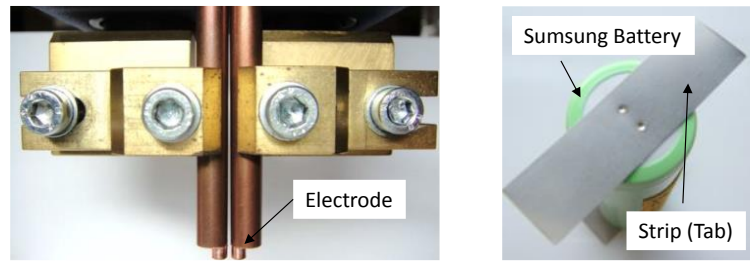

Fig. 1. Position in each component.

Configurations used in simulation are demonstrated in Fig. 2, where conditions, boundaries and material properties are assigned accordingly.

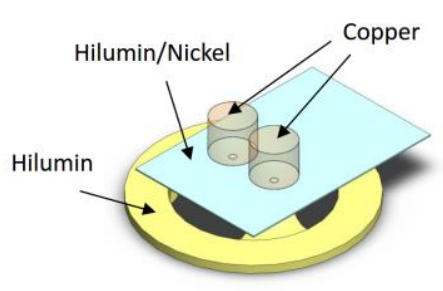

a) Positive side

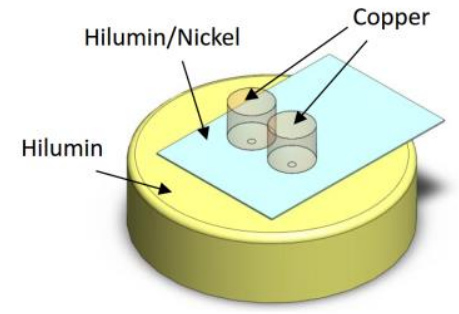

b) Negative side

Fig. 2. Material setting in Simulation.

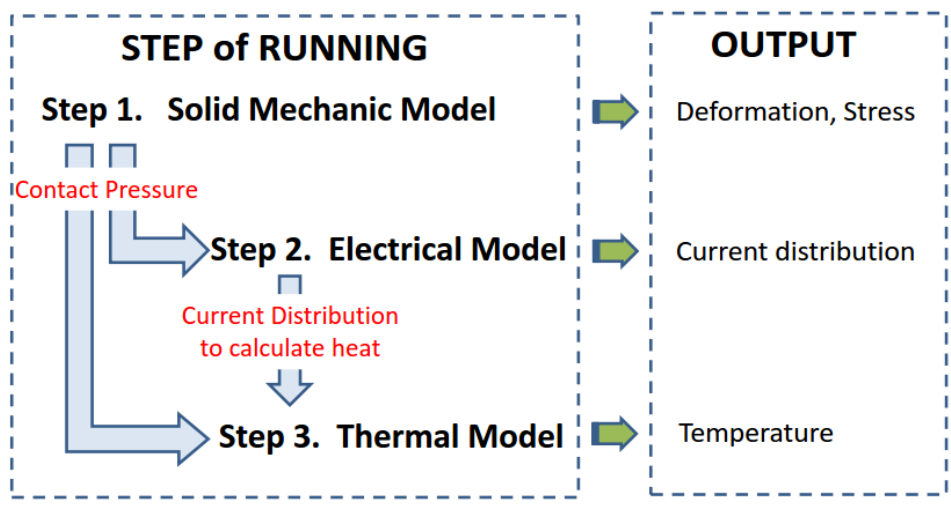

Fig. 3. Simulation running step.

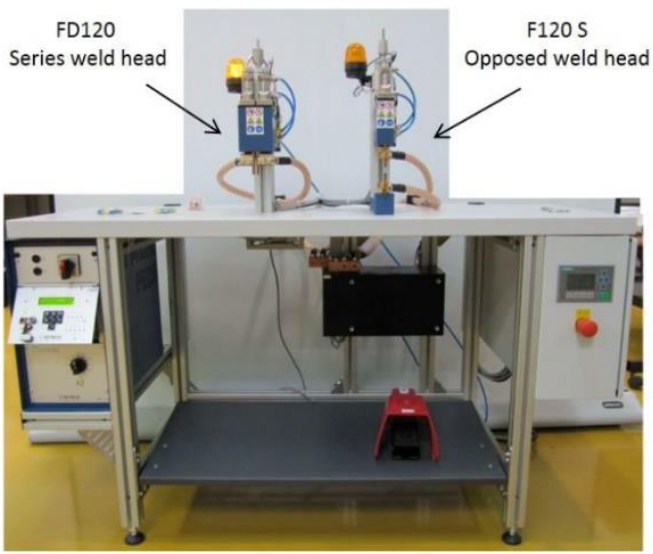

Fig. 4. Welding machine including series and opposed weld heads [4]. 


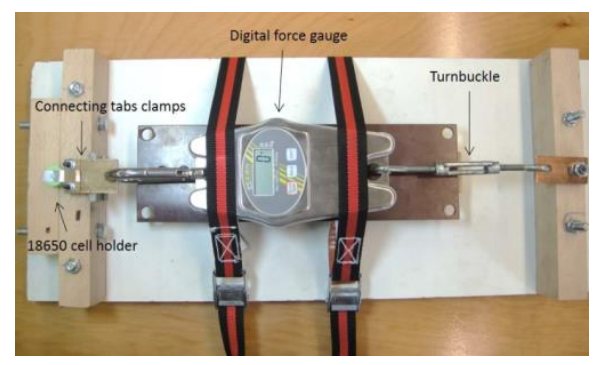

Fig. 5. Devices setup for mechanical strength test.

\section{Simulation and Experimental Setups}

\subsection{Simulation Setup}

All simulations in this work were carried out with COMSOL Multiphysics 5.0 simulation software and run on a computer using Windows 7 64-Bit operating system with Intel Core 2 Quad CPU Q8400@ 2.66GHz $2.67 \mathrm{GHz}$ processor and $8 \mathrm{~GB}$ of RAM. The computer is considered not suitable to run complicated model including solid mechanic, electrical and thermal physics at the same time. Owing to limit of performance of the computer, the model of the welding is divided into three steps, where each step provides its own outputs as shown in Fig 3. In Step 1, solid mechanic model is used to find the contact pressure between materials such as an electrode with an electric strip and an electric strip with the battery case both on positive and negative terminals. The contact pressure distribution obtained from Step 1 will be used in Step 2 and Step 3. In addition, Step 1 can also give outputs that are deformation and mechanical stress in work-pieces. In Step 2 , electric contact as function of contact pressure from Step 1 is used for the calculation of electrical conductivity of contact surface in the electrical model. This model can calculate current distribution inside of the work-pieces that will be used to calculate heat generation in the thermal model in Step 3. In Step 3, the thermal model uses not only the current distribution from Step 2 to calculate the heat generation, but also contact pressure contact from Step 1 to calculate the thermal conductivity between contacts. Therefore, Step 3 can calculate temperature distribution.

\subsection{Experiment Setup}

Figure 4 shows the welding machine (Miyachi FD120) composes of a series (parallel gap) spot weld head (left) and opposed spot weld head (right). The series weld head is used in this work.

In order to measure the mechanical strength of the welded contact between the connecting tab and the 18650 battery cell, a digital force gauge with a maximum range of $2000 \mathrm{~N}$ is mounted on the fixture shown in Fig. 5. At the left end, a battery cell is fixed and the connecting tab is clamped. The gradual increasing of tensile force was applied via a turnbuckle fixed at the right end. Due to the limitation of equipment utilized in this experiment, the turnbuckle was turned by hand and in the meanwhile a video camera was used to capture a precise observation of the maximum force value detected by the digital force gauge. It is noted that the turning speed of a turnbuckle cannot be consistently controlled by hand in the current experiment; a screw motor may be used for higher precision in the future.

In order to measure electric current and voltage during welding, a Hioki Clamp on Meter AC/DC HiTester 3285 used to detect the electric current flow was set in AC mode with a current range of $0-2000$ A and was connected to an oscilloscope (HAMEG HMO 1024). The voltage was measured by connecting the oscilloscope direct to the electrodes. Figures 6 and 7 illustrate an example measurement from experiment. Moreover, the voltage profile in Fig. 6 was filtered to use in the simulation to calculate current distribution in each work-piece. However, current profile from simulation in Fig. 7, which flows totally through the electrode, is very complicated to know because surface roughness is not constant during welding process but it depends on temperature. The welding process completes in less than $0.1 \mathrm{sec}$, resulting in the incapability to measure the rapid temperature change. The simulation is then used to aid the estimated temperature distribution. 


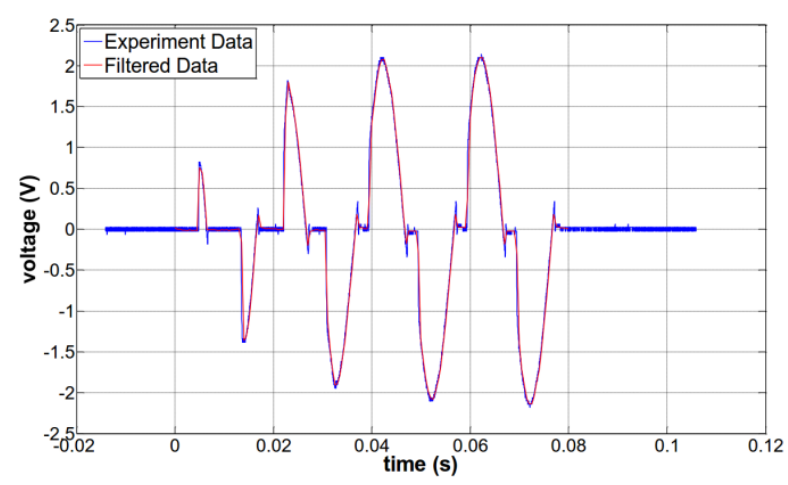

Fig. 6. The voltage profile from experiment and after filtered.

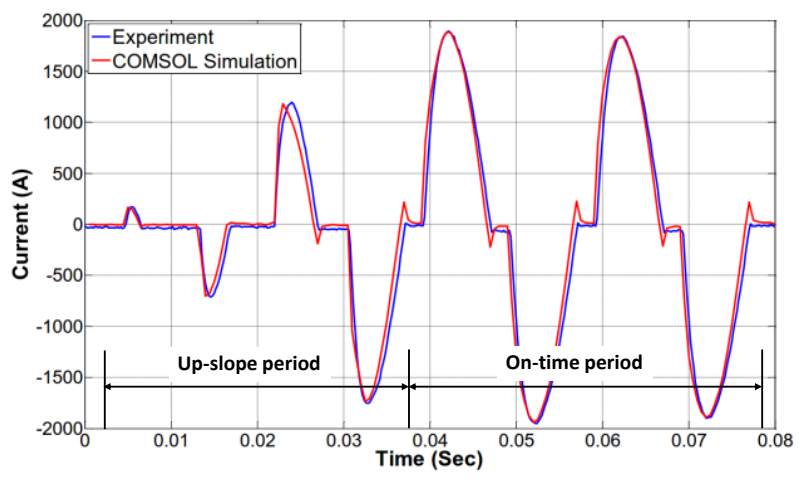

Fig. 7. Comparison of current between experiment and simulation using surface roughness as function of time.

\subsection{Surface Roughness Calibration}

AC/DC Module and Heat Transfer Module in COMSOL require surface roughness value as function of temperature during welding. Detailed technique to determine the surface roughness value as function of temperature is represented in this work. A voltage profile is measured from an experiment is inputted to COMSOL model. After that, COMSOL is run to get current profile and compared with current profile from the same experiment. If current profile from COMSOL is not equal to the experiment, surface roughness value will be adjusted until their current profiles are as close as possible. However, it cannot compare all of them in every period; only periods having the peak current are compared. In this work, only one case study for Hilumin ${ }^{\circledR}$ tabs on the positive terminal is simulated to find temperature during welding due to the time limit. For parameter of the case study, maximum supply voltage is $2.5 \mathrm{~V}$, Up-slope is 2 cycles, On-time is 2 cycles and pressing force of each electrode is $20 \mathrm{~N}$. The detailed procedures for adjusting the surface roughness are described as following;

Step 1 - Filter the voltage and current profiles from the experiment to reduce noise owing to the measurement. Meanwhile, only the period of welding is captured to reduce inadequate data. To reduce noise of measurement, the voltage profile from experiment was filtered by MATLAB.

Step 2 - Select peak of voltage of each period as shown in Fig. 8.

Step 3 - Each of the peak voltages is inputted in AC/DC Module in COMSOL one by one to run and check the peak current outputs from COMSOL with experiment. If the currents are not close to that of experiment, the surface roughness will be varied until the currents are as close as possible; it can be shown in Fig. 7. Therefore, the surface roughness as function of time can be plotted in Fig. 9. In sum, using the surface roughness from Fig. 9, electric current in simulation can be calculated and has the tendency near the result of experiment. 


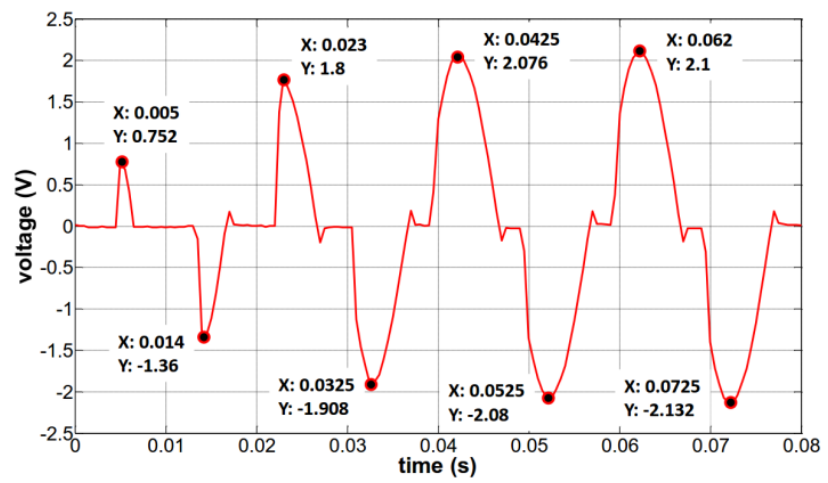

Fig. 8. Peak voltage of each period for a case study.

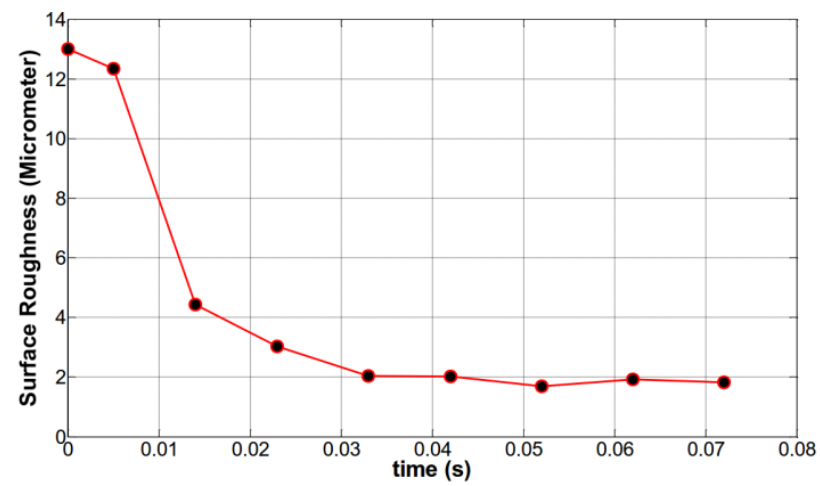

Fig. 9. The surface roughness as function of time for a case study.

\section{Results and Discussion}

\subsection{Electrode Tip Geometry}

There are three electrode tip geometries as shown in Fig. 10. To investigate their behaviors during and after welding, both simulations and experiments are used. The results obtained from the simulations showing deformation, electric current density, and temperature distribution occurred on work-pieces with different electrode tip geometries are illustrated in Fig. 12, Fig. 13, and Fig. 14 respectively. Meanwhile, oxidation and sparking at the head of the electrodes were analyzed by observation for the experiments.

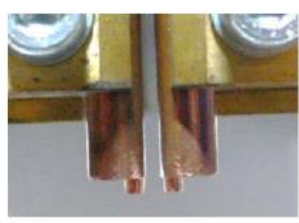

a) Flat tip
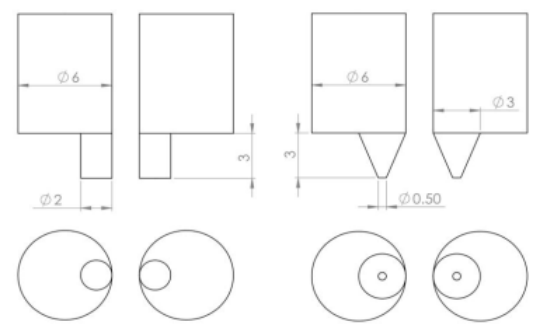

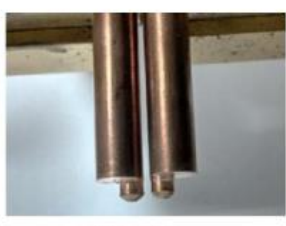

c) Round tip

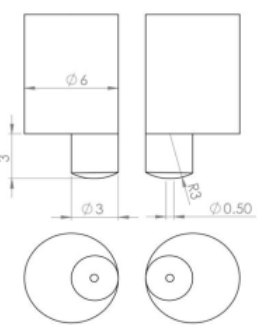

Fig. 10. Three electrode tip geometries. 


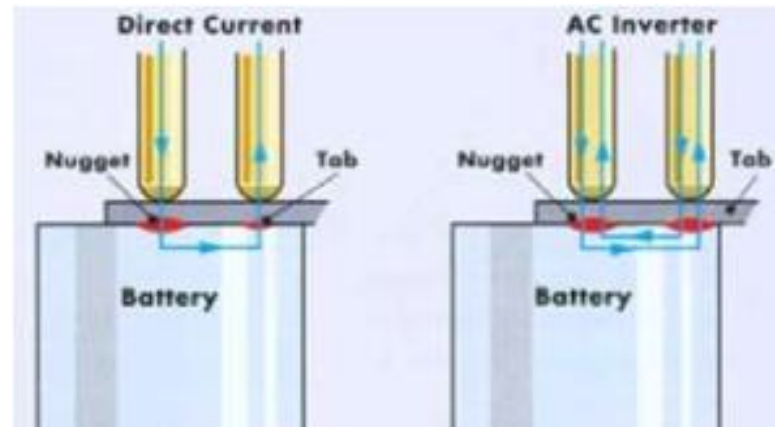

Fig. 11. Current flow comparison between DC and AC Input [5].

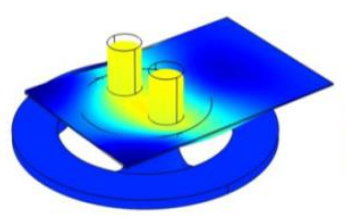

(a) Flat electrode tip

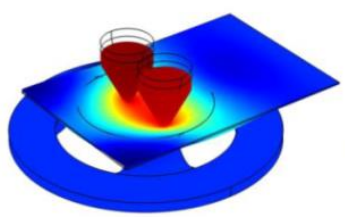

(b) Tapered electrode tip

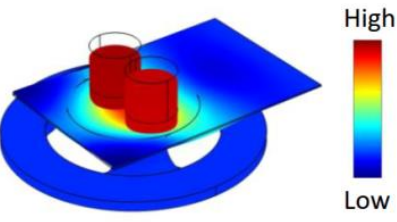

(c) Round electrode tip

Fig. 12. Deformation of work-piece comparison between different electrode geometries.

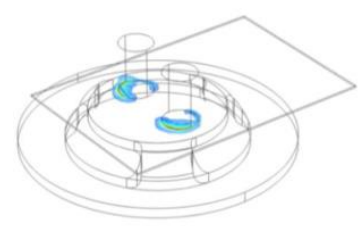

(a) Flat electrode tip

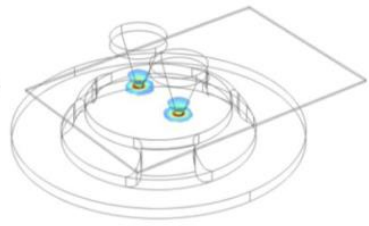

(b) Tapered electrode tip

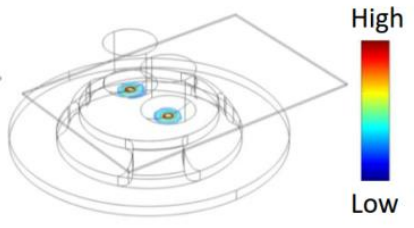

(c) Round electrode tip

Fig. 13. Current distribution around the end of electrode tips.
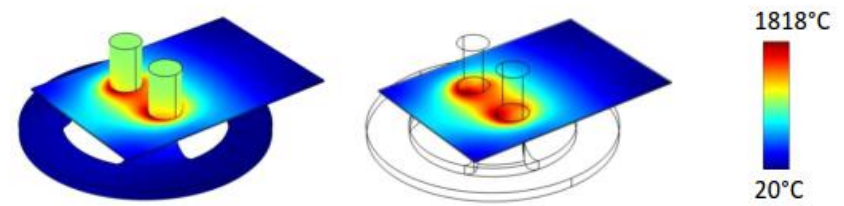

(a) Flat electrode tip
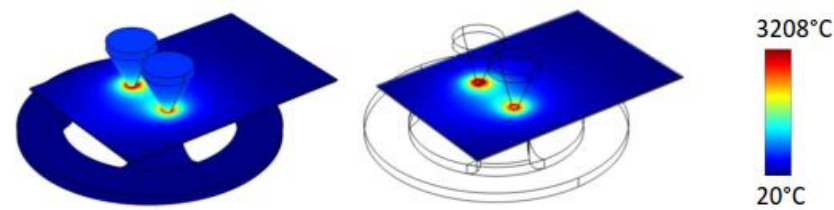

(b) Tapered electrode tip
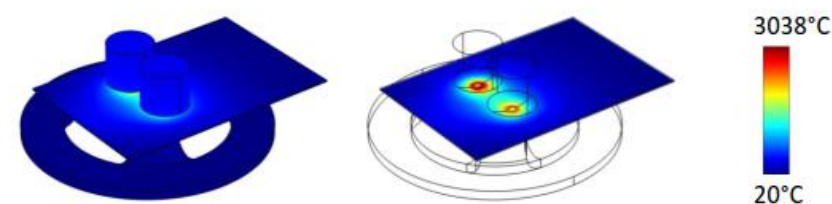

(c) Round electrode tip

Fig. 14. Temperature distribution on work-pieces. 


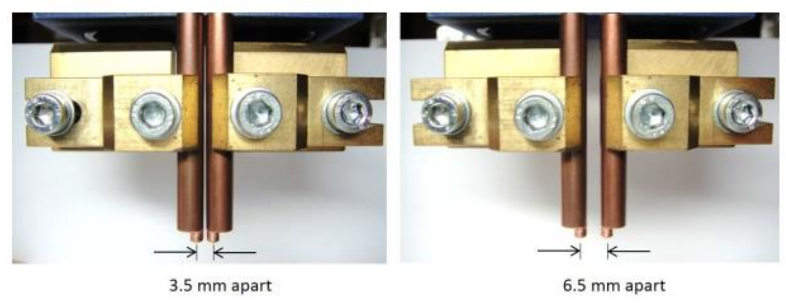

Fig. 15. Different electrode distances.

Two simulation setups were tested for comparison purpose, i.e. using DC (Direct current) and AC (Alternating Current) input. DC input has the advantage for being simple method and using less simulation time. The DC (Direct Current) input was applied to one electrode, and ground connection to another electrode. The heat generated at the end of one electrode tip is relatively greater than another tip due to the fact that there is only one direction of current flow as illustrated in Fig. 11 and Fig. 14. The uneven heat distribution on electrodes causes one electrode tip to wear faster than the other. On the other hand, with AC (Alternating Current) input, the current flows through both electrodes alternatively which helps avoiding asymmetrical wear of the electrodes. This behavior suggests AC input is preferred for series weld heads. According to the results from simulations and experimentations, the effects for different electrode tip geometries can be summarized as following:

\subsubsection{Flat electrode tip}

Even though the welding electrode with off-center flat tip design is the easiest shape to manufacture due to its simple geometry, it does not produce effective weld results. Not only does the relatively large tip diameter yield inadequate deformation on work-pieces as shown in Fig. 12, but also contributes to a ring shape of current density distribution along the edge of electrode tip. Moreover, as a consequence of the geometry of positive cap reinforcement, it can be seen in Fig. 13 that there is higher current density along the outside edges of electrode tips where more reaction force (pressure contact) from the positive cap are presented causing uneven heat generation. Excessive diameter of electrode tip also conducts heat dispersion in the region between two welding electrodes as illustrated in Fig. 14, which results in a poor weld contact quality. Hence, this electrode shape is not suitable for spot welding on this battery cell.

\subsubsection{Tapered electrode tip}

With a tapered electrode tip, small welding points can be formed between work-pieces surfaces which contribute to higher current density flow at the welding point as shown in Fig. 13. Consequently, higher temperature can be generated developing a stronger welding contact. Although the tapered shaped electrode tips promote a better weld contact quality, it happens that the electrode tips get worn away quickly after several welding processes.

\subsubsection{Round electrode tip}

From trial welding experimentation, it appears that the round-shaped electrode tips with small flat tip diameter offers similar spot weld results when compared to the tapered-shaped electrode tips, but with a moderate wear of electrode tips after the number of welding processes. Thus, it is more preferable than tapered electrode tips for mass production spot welding. However, the round-shaped tips are relatively more difficult and required more time to make them manually by hand. Accordingly, for a mass production spot welding, it is recommended to manufacture the welding electrodes with the CNC (Computer Numerical Control) machining to obtain a uniform geometry of electrode tips.

\subsection{Distance between Two Welding Electrodes}

The deformation of work-pieces and welding electrodes with mounting distance of $3.5 \mathrm{~mm}$ (left) and $6.5 \mathrm{~mm}$ (right) on the negative terminal are demonstrated with real pictures in Fig. 15 along with their 3D and 2D 
models in Fig. 16. It can be noticed that there is comparatively higher deformation occurred with a shorter electrode mounting distance of $3.5 \mathrm{~mm}$. Similar to the case of positive cap geometry described in a previous section, the regions at the center far from the edge of the negative terminal are subject to lower support from the battery housing, resulting in greater deformation. Not only does excessive deformation of work-pieces mean greater damages of battery components, but can also lead to poor strength of weld contact, which will be later explained in Fig. 25 of later section. Furthermore, from the welding experiments with different values of mounting distance between electrodes, it happens that the differences in electrical resistance of the connecting tabs material between $3.5 \mathrm{~mm}$ and $6.5 \mathrm{~mm}$ of electrode distances are minimal that contribute to insignificant changes in electric current flow between two settings as shown in Fig. 17. Even though a little variation in welding distance has almost no effect on electric current, it is still recommended that the weld spots should be located in the regions far away from the center of the battery cell in order to avoid redundant deformation. Considering the least processing time for mass production spot welding in which both positive and negative terminal of battery cells may be consecutively welded with connecting tabs to create battery packs, it is more efficient for the electrodes mounting distance to remain unchanged (e.g. at $3.5 \mathrm{~mm}$ apart). Since multiple-spots welding on one battery terminal are likely to be conducted in favor of better weld connections, Fig. 18 suggests some alternatives for positioning weld spots on a connecting tab even with a short mounting distance between two electrodes.

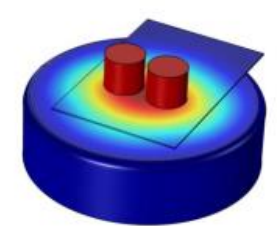

Electrodes are $3.5 \mathrm{~mm}$. apart

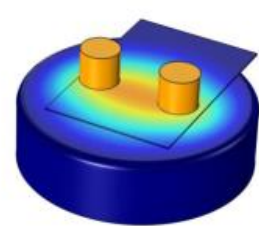

Electrodes are $6.5 \mathrm{~mm}$. apart

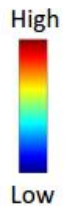

(a) 3D models.

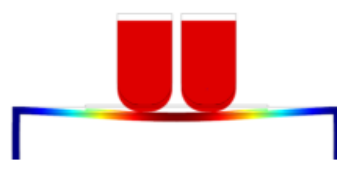

Electrodes are $3.5 \mathrm{~mm}$. apart

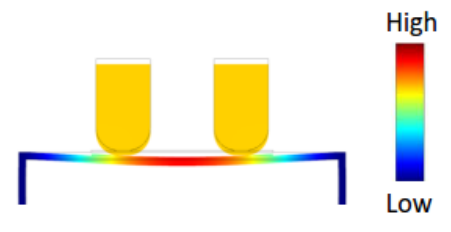

Electrodes are $6.5 \mathrm{~mm}$. apart

(b) $2 \mathrm{D}$ models.

Fig. 16. Deformation due to different electrode distances.

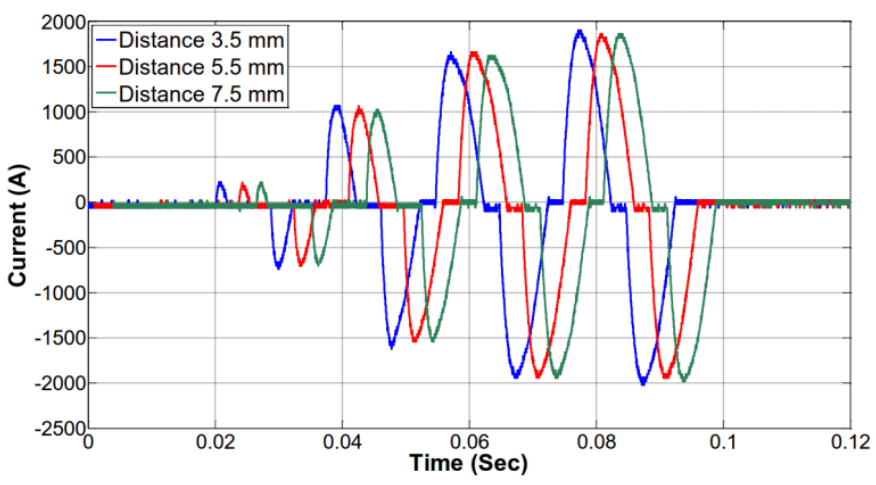

Fig. 17. Current comparison between difference settings of distance between electrodes. 


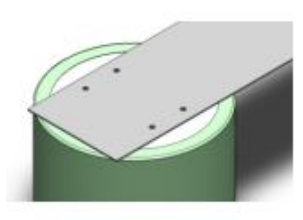

2 times

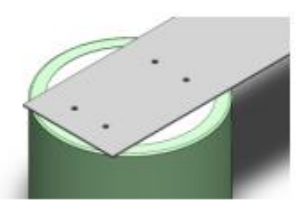

2 times

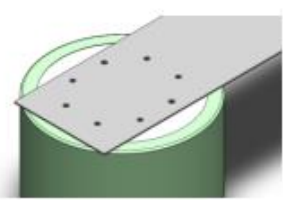

4 times

Fig. 18. Various positioning alternatives of weld spots for multiple-spots welding with short distance between electrodes on the negative terminal.

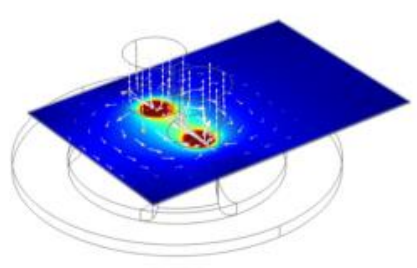

Connecting tab without slot
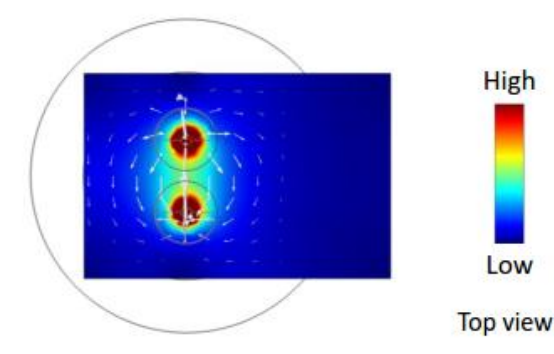

Top view

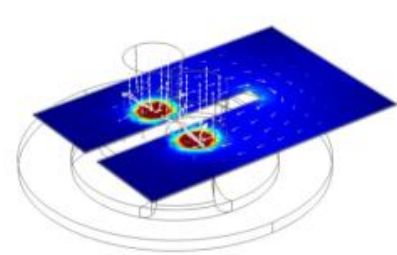

Connecting tab with slot
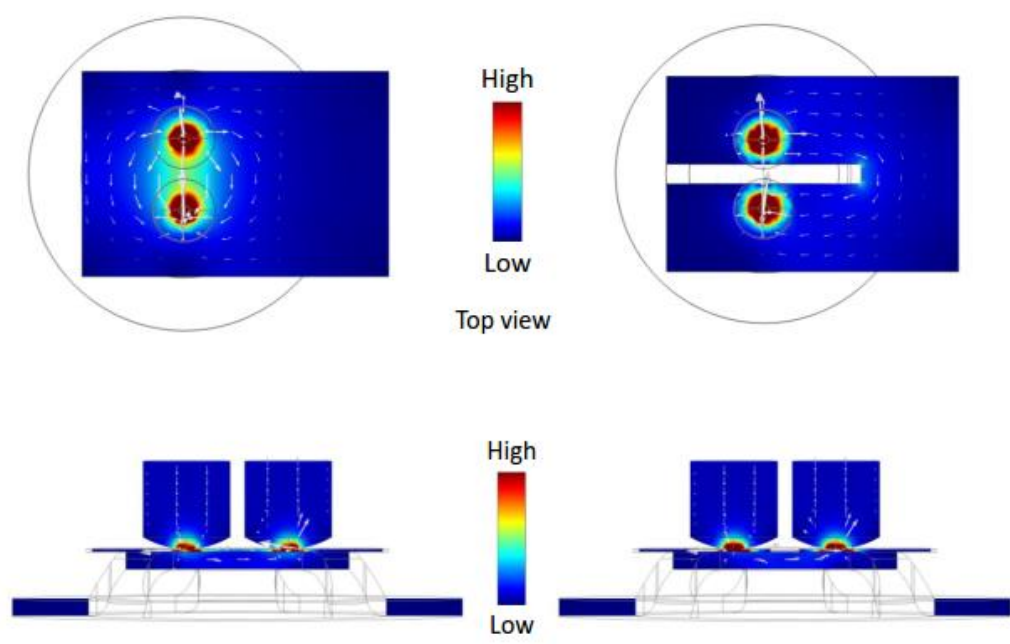

Front view

Fig. 19. Electric current distribution comparison between models comprising connecting tabs with and without slot.
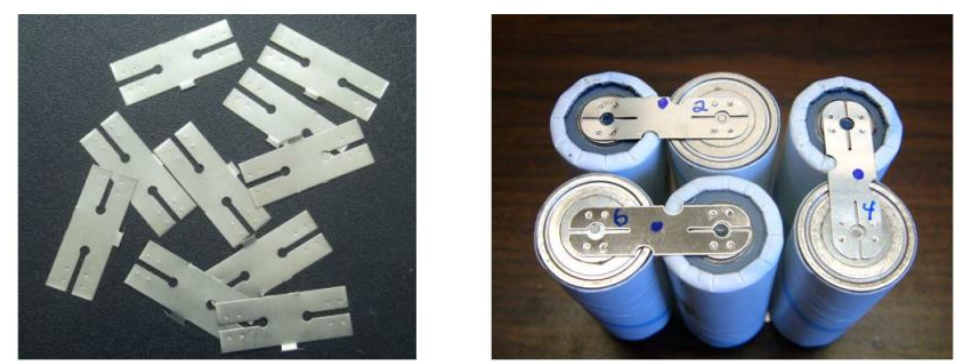

Fig. 20. Design alternatives for connecting tab with slot $[6,7]$. 


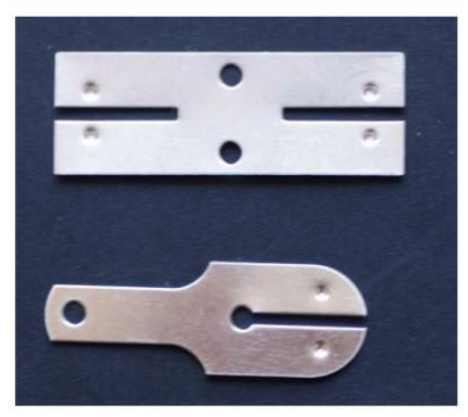

Fig. 21. Examples of connecting tabs with slot and prominent bumps [8].

\subsection{Connecting Strip Design}

Figure 19 illustrates simulation results comparing the electric current flow paths between the left model with a normal connecting tab without slot, and the model on the right side consisting of a connecting tab with slot. Due to the fact that electric current tends to flow along the path that has the lowest resistance, it can be seen in the front view of Fig. 19 that there are flows of current along the upper layer of work-pieces (connecting tab). Moreover, the current also scatteringly flows around two welding electrodes as shown in the top view of Fig. 19. These flow behaviors results in a dispersing of heat on the connecting tab, as a consequence a desired maximum temperature cannot reach. Thus, a higher input current is required in order to attain a melting temperature of material creating welded spots. Alternatively, connecting tabs with slot at the middle are being used in the market in favor of controlling the current flow paths to pass through small electrode tips down to the contact points between connecting tab and battery terminal without flowing along the middle region between two weld spots. As a result, the welded spots are easier to obtain due to small region of heat are being generated and a higher maximum temperature can be reached. However, it can be noticed that there are also some current flows around the slot contour due to insufficient resistance along the path, this can be avoid by a modification of slot geometry to increase the resistance as shown in Fig. 20. For connecting tabs with thickness higher than $0.15 \mathrm{~mm}$, higher input voltage/current is required. Other than using connecting tab having slot, creating prominent bumps on the tabs as shown in Fig. 21 could easily promote small spots of current concentration. As a result, a decent weld contact can be formed at a lower input voltage current setting and the electrode life can be prolonged. With the same amount of current supply, using a connecting tab having slot at the middle would yields a better weld contact than the one without slot. In other words, to equally achieve decent welded contacts strength, the connecting tab with slot required less input current than the normal one. Nevertheless, creating slot on the connecting tab required additional manufacturing process, consequently higher production time. Therefore, one has to compromise the cost, the time effective and the satisfactory weld quality.

\subsection{Connecting Strip Material}

After obtaining the data from experimentations of spot welding the positive terminal of battery cells with Hilumin ${ }^{\circledR}$ and Nickel tabs respectively, the filtered voltage profiles were used as input voltages for simulation in COMSOL. The integrated current flows within two models, one composed of connecting tab material made of Hilumin ${ }^{\circledR}$ steel grade (UNS G10060), and another model with Nickel 200 (UNSN02200) connecting tab, are compared as shown in Fig. 22. It can be seen that the maximum amplitude of the current profile of a model with Nickel connecting tab is slightly higher than that of a model with Hilumin ${ }^{\circledR}$ tab, which owes to the fact that the electric conductivity of Nickel 200 is also relatively higher than that of Hilumin ${ }^{\circledR}$. The maximum temperatures of the Hilumin ${ }^{\circledR}$ and Nickel tabs occur during spot welding are also investigated via simulation and illustrated in Fig. 23. The temperature profiles of both materials rapidly rise at first and then keep increasing gradually until reaching the maximum temperatures, which are close to the melting point of ductile iron and pure nickel with values approximately around $1,150^{\circ} \mathrm{C}$ and $1,450^{\circ} \mathrm{C}$ respectively [9]. Moreover, as seen in Fig. 24, the temperature of both Hilumin ${ }^{\circledR}$ and Nickel tabs rapidly drop from the maximum temperature to a point below $100^{\circ} \mathrm{C}$ after a few seconds passed. This behavior is advantageous to a series-spot welding process where the connecting tab and battery cell can be continuously welded at multiple spots in order to increase the contact area. Consequently, the electrical conductivity and mechanical strength 
of the weld contacts are improved. However, the results of electric current and temperature obtained from simulations are only used to study the different behavior between Hilumin $\AA$ and nickel material. The actual values of current and temperature occurred in the real work-pieces are likely to depend to many other influencing factors such as heat convection coefficient, thermal radiation, surface roughness of materials etc. Furthermore, in order to determine either Hilumin ${ }^{\circledR}$ or pure nickel is more suitable as connecting tab material for spot welding, the measurement of contact resistance and mechanical strength are necessary.

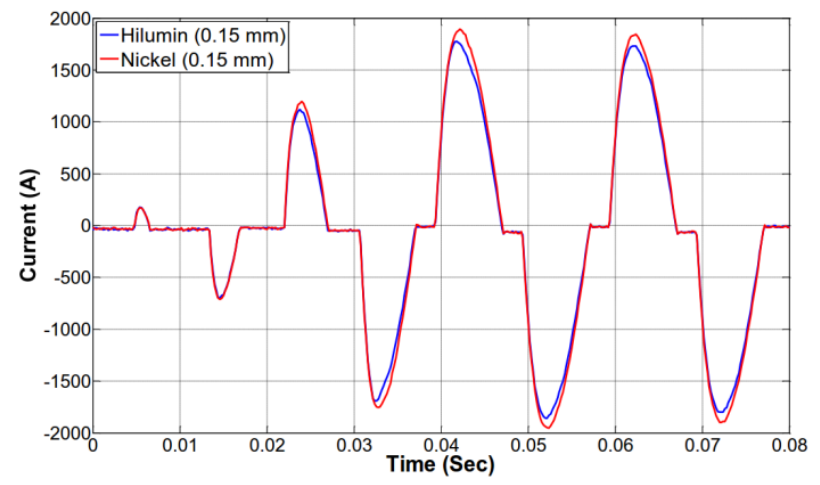

Fig. 22. Current comparison between Hilumin ${ }^{\circledR}$ and Nickel tabs from simulation.

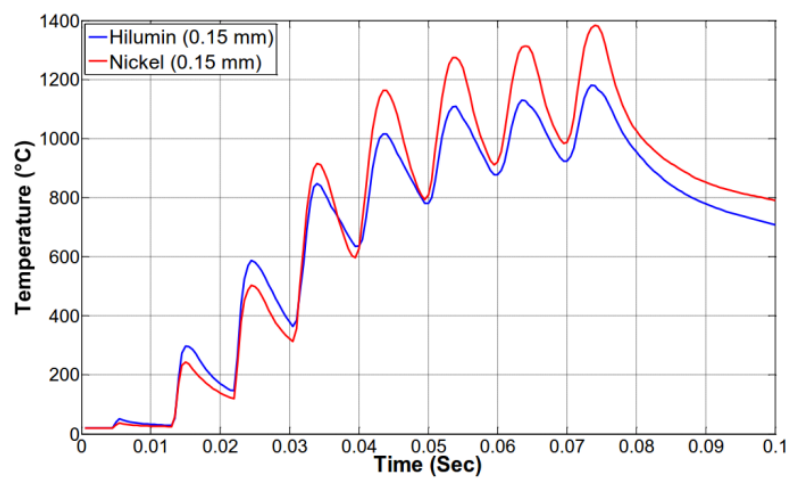

Fig. 23. Maximum temperature comparison between Hilumin $®$ and Nickel tabs from simulation.

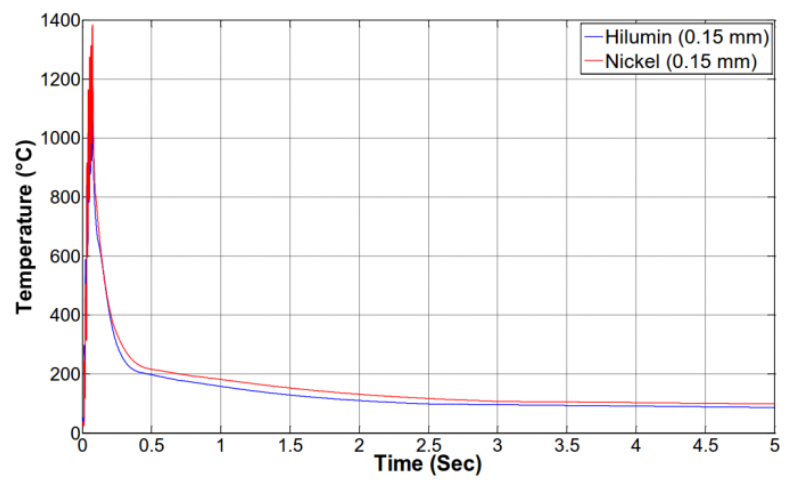

Fig. 24. Temperature drop of Hilumin $®$ and Nickel tabs. 

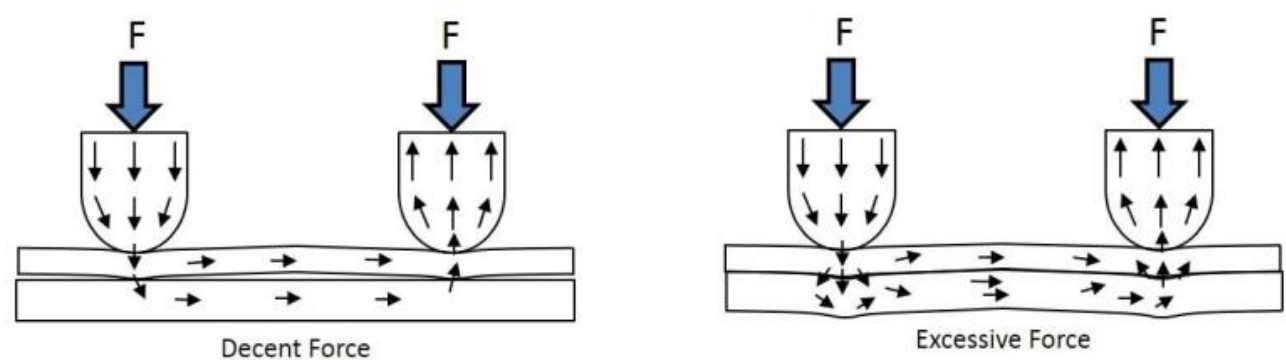

Fig. 25. Current flows comparison for different amounts of welding force applied.

\subsection{Welding Force}

Generally, the higher the electrode welding force is, the greater the deformation is on the work-pieces. However, for the spot welding between Hilumin ${ }^{\circledR}$ tabs of $0.1 \mathrm{~mm}$ thickness and $0.3 \mathrm{~mm}$ thickness, the change in deformations for different amount of welding force applied could not be easily observed due to the present of good supporting base underneath welding tabs. On the other hand, in the actual case of welding a connecting tab on a battery cell, when too much force is being applied on the negative terminal of a cell, especially at the center area, high deformation occurs as a result of having lower support reaction from the battery casing compared to the position further away from the center. This consequently affects the current flow paths as illustrated in Fig. 25. Too much deformation of work-pieces leads to a large contact area between the surfaces of upper and lower work-pieces. This allows the current to flow along various paths rather than a small spot causing a wide range of temperature distribution within work-pieces. Without reaching the melting temperature of materials, this results in either contacts with poor strength or unbonded contact. Nevertheless, an electrode welding force should not be too low, since a weak connection at welding contact during a welding process can produce a light arc which causes metal sparking. Hence, a decent amount of force that creates a good touching point contact is preferred for an ideal spot welding result.

\subsection{Welding Time}

Figure 26 shows the examples of spot welding results between $0.1 \mathrm{~mm}$ thick and $0.3 \mathrm{~mm}$ thick Hilumin ${ }^{\circledR}$ tabs with three different welding time settings. While the shortest welding time is generally preferable to achieve the shortest overall production time, a spot welding with too short welding time can however lead to inadequate deformation which consequently forms a poor contact. On the contrary, excessive welding time not only can causes redundant deformation, but can also generate too much heat giving rise to metal flame, which results in burnt mark in the middle area between two weld spots as well as high wear of electrode tips. This situation nevertheless can also be avoided by creating a slot in the middle of the tab between welding points in order to control the current flow path, as previously illustrated in Fig. 19. Proper welding time setting would yield a good welding contact with decent deformation and without having burn marks on workpiece surface. In practice, the trial welding should begin with the shortest welding time setting, and the ontime period should be step by-step increased until satisfactory weld results are achieved.

\subsection{Maximum Supply Voltage}

The spot welding results between Hilumin ${ }^{\circledR}$ tabs with different maximum supply voltage settings are shown in Fig. 27. The effects of welding voltage level on work-pieces are somewhat similar to the effects from welding time setting but with more pronounced impact. Low stage of maximum voltage allows only small amount of power releases to the welding electrodes and subsequently workpieces. In case that the heat generated is too little, not only is inadequate deformation of the Hilumin ${ }^{\circledR}$ tabs created developing poor contact pressure, but also the melting temperature of work-piece material may not reach causing poor or unboned weld contacts. Although longer welding time maybe set in order to achieve the targeted temperature, it can also lead to unwanted effects due to excessive welding time as formerly shown in Fig. 26. In contrast, at higher maximum voltage stage, shorter welding time is required due to the fact that greater amount of electric power can be generated which generally promotes a stronger weld contact. However, as shown in Fig. 27, applying too much power to the weld contacts can cause redundant deformation and also excessive 
heat generated which creates dark circular burnt marks on the work-piece surface around weld spots as well as at the middle area between two welding points resembling the effects due to high welding time previously described. Under high humidity environment, these burnt marks can lead to an electrochemical corrosion through oxidation of iron, and eventually develops into rust. Thus, a decent supply voltage that promotes adequate mechanical strength of weld contacts without causing burnt marks is suitable for applications that require a long lifespan connection of a battery pack. Similar to welding time setting, it is recommended to start with the lowest maximum supply voltage stage and current strength setting for trial welding. If the satisfying weld results are still not obtained at the maximum current strength setting, the maximum voltage value should then be increased to the higher stage. Additionally, performing multiple-spots welding creating higher number weld spots between work-pieces is also recommended to promote a stronger connection results.

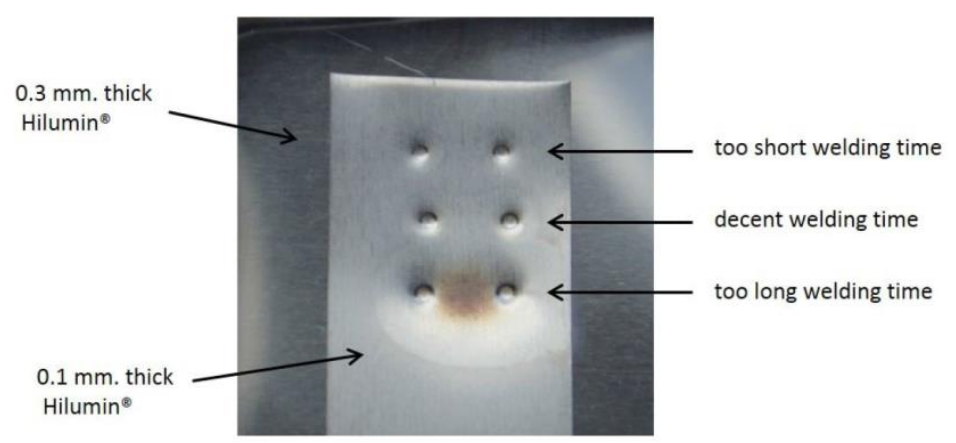

Fig. 26. Welded spots comparison for different welding time settings.

\section{Optimized Parameter Range}

After having the results from the studies of the relevant welding parameters via simulations and experimentations previously described, the optimization for parameter ranges of maximum supply voltage, welding, time and welding force by spot welding connecting tabs on actual battery cells were further conducted with suitable parameters setting that had already been determined as follows:

- Electrode tip design Round tip electrode with $3 \mathrm{~mm}$ offsetted diameter and $0.5 \mathrm{~mm}$ tip diameter

- Distance between welding electrodes $3.5 \mathrm{~mm}$ apart for welding on positive terminal, and $6.5 \mathrm{~mm}$ apart for welding on negative terminal

- Connecting tab design and material Hilumin ${ }^{\circledR}$ tabs of $0.1 \mathrm{~mm}$ thick and $10 \mathrm{~mm}$ wide, without slot in the middle

By obtaining the results from performing trial spot welding between $0.1 \mathrm{~mm}$ in thickness and $0.3 \mathrm{~mm}$ in thickness of Hilumin ${ }^{\circledR}$ tabs, the value range of maximum supply voltage, welding time, and welding force can be narrowed down for conducting the actual spot welding $0.1 \mathrm{~mm}$ thick Hilumin ${ }^{\circledR}$ tabs on both positive and negative terminals of the Samsung INR18650-15L Li-ion battery cells. The followings are the bounded values for each parameter to be varied;

- Maximum supply voltage stage 2.0 and 2.5 Volts

- Welding time Upslope 1 and 2 cycles, On-time 1 and 2 cycles

- Welding force 30 and $40 \mathrm{~N}$

The varied values were combined into 14 parameters sets for conducting experiments. Each parameter set was tested twice adding up to a total number of 28 battery cells used. Following the welding processes, the electrical resistance and mechanical strength of each weld contacts were measured in order to compare and determine the suitable values of maximum supply voltage, welding time, and welding force that yield the optimal welding results. The results for the measurement of contact resistance and maximum shear force for welding Hilumin ${ }^{\circledR}$ tabs on positive terminal and negative terminal of Samsung INR18650-15L Li-ion battery 
cells can be found in Table 1 and Table 2 respectively. Examples of spot welding results between Hilumin ${ }^{\circledR}$ connecting tabs of $0.1 \mathrm{~mm}$ thick and Samsung INR18650-15L Li-ion battery cells are illustrated in Table 2 . It can be seen in both results tables that the differences of contact resistance measured on the weld contacts using different welding parameter sets are very minimal. Since the electrical resistances were measured between the center points on each side (terminal) of battery cell, the value of resistance measured on the contacts weld on negative terminal are slightly higher than that on positive terminal due to greater welding distance between electrodes. According to minimal differences in electrical resistances of the weld contacts, the value of mechanical strength is then considered as a deciding factor to distinguish the weld quality. The parameters setting in ranges highlighted with green color in Table 1 and Table 2 are the suitable values of maximum supply voltage, welding time, and welding force, which can resist to more than $200 \mathrm{~N}$ of load in the shear direction. The parameter combinations that fall in the blue-highlighted zone produce the weld contacts that are too weak or unboned as indicated in Fig. 28. Although some of the parameter combinations with a higher maximum supply voltage setting of $2.5 \mathrm{~V}$ (pink-highlighted zone) create weld contacts that can handle to a little higher shear force, there were welding splashes generated during welding process due to excessive current supplied. As a result, the electrode tips were rapidly eroded. Furthermore, too much heat generated also causes burn marks around the weld spots, which can develop into rust in the long run under high humidity conditions. In practice, the welding parameters range in other environment may however vary from value ranges suggested in this work due to some uncontrollable factors in the experiments such as welding electrodes wear, heat convection, surface roughness of workpieces material etc.

Table 1. Measurement of contact resistance and shear force for positive terminal welding.

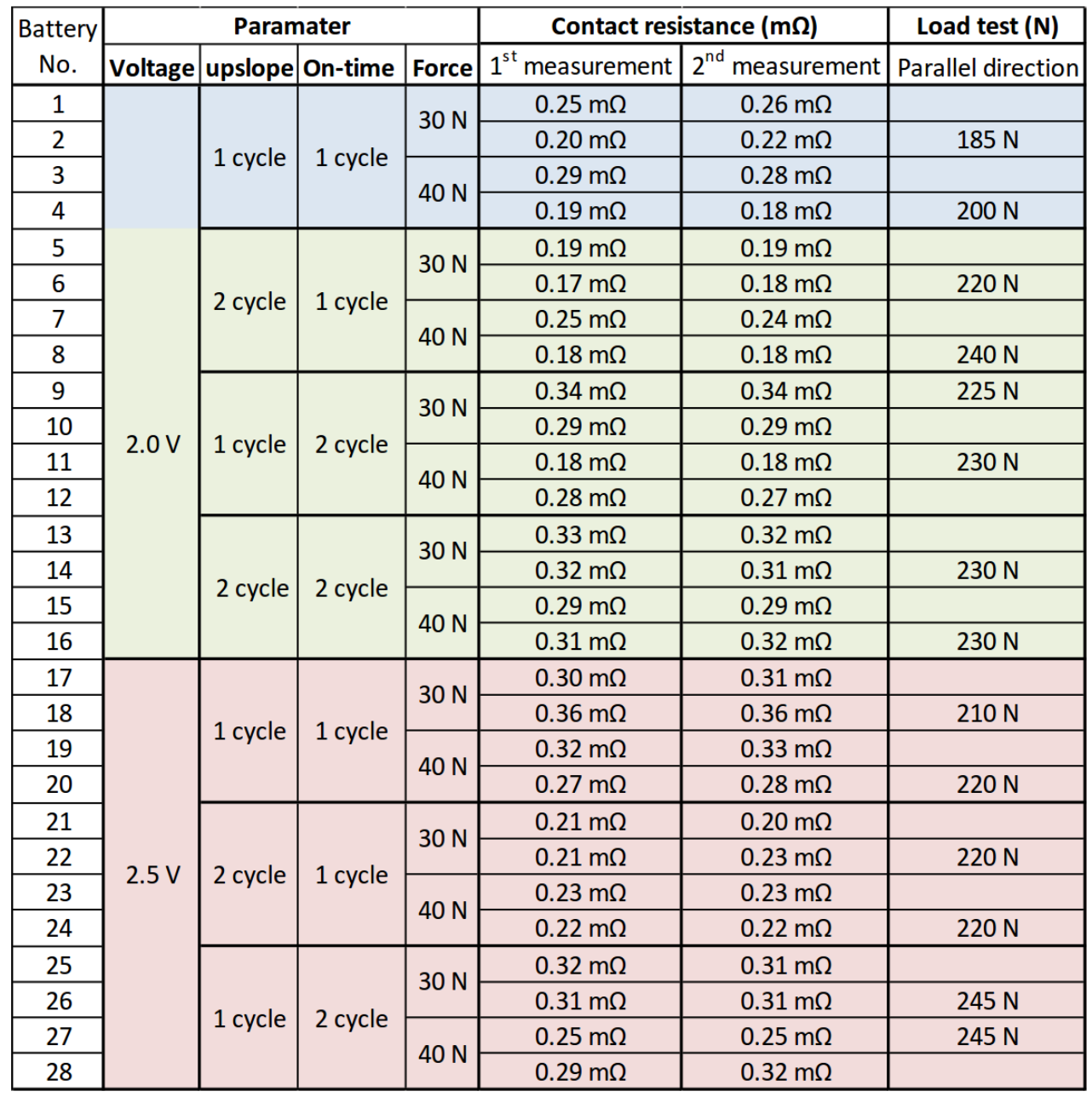


Table 2. Measurement of contact resistance and shear force for negative terminal welding.

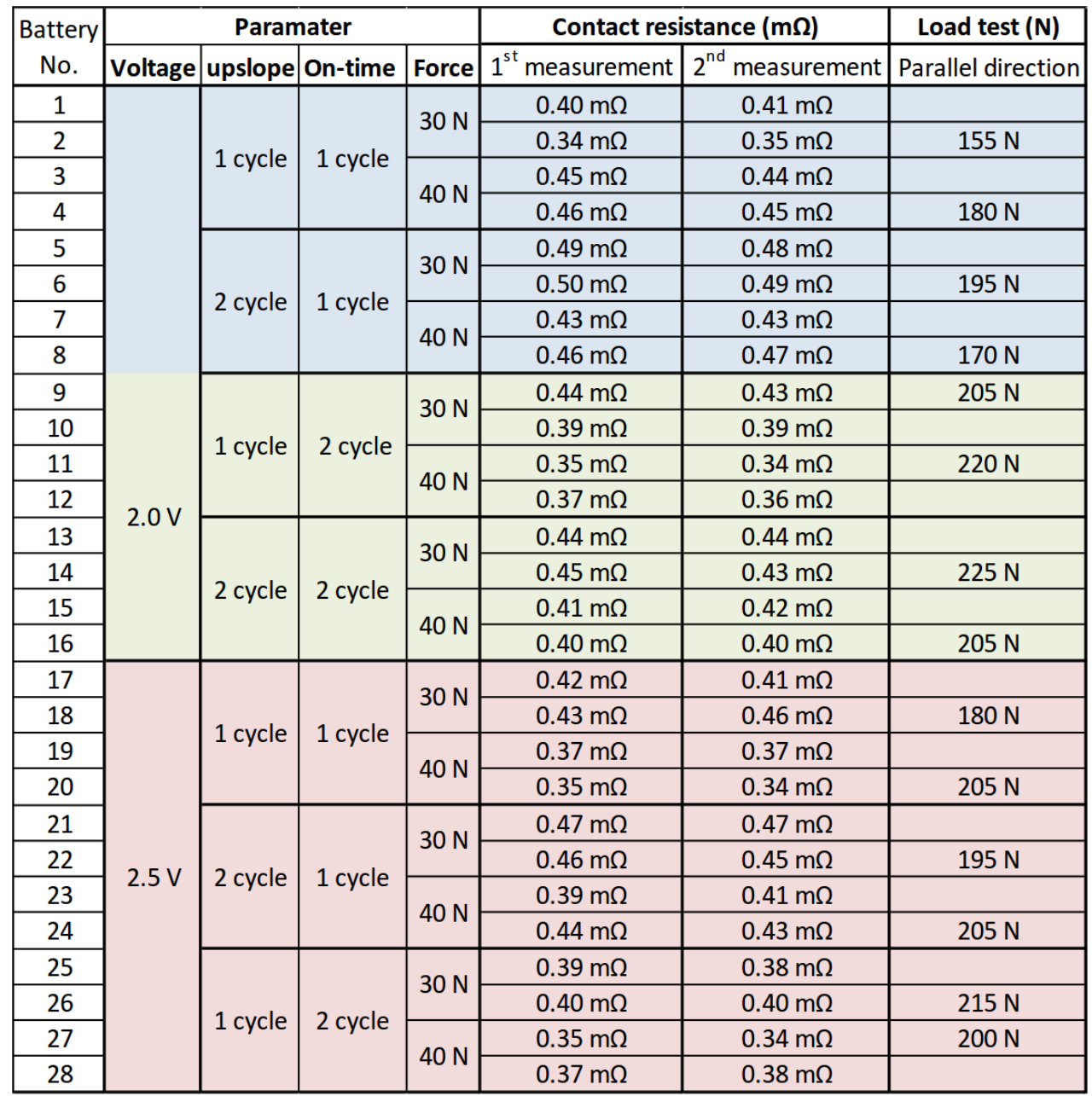

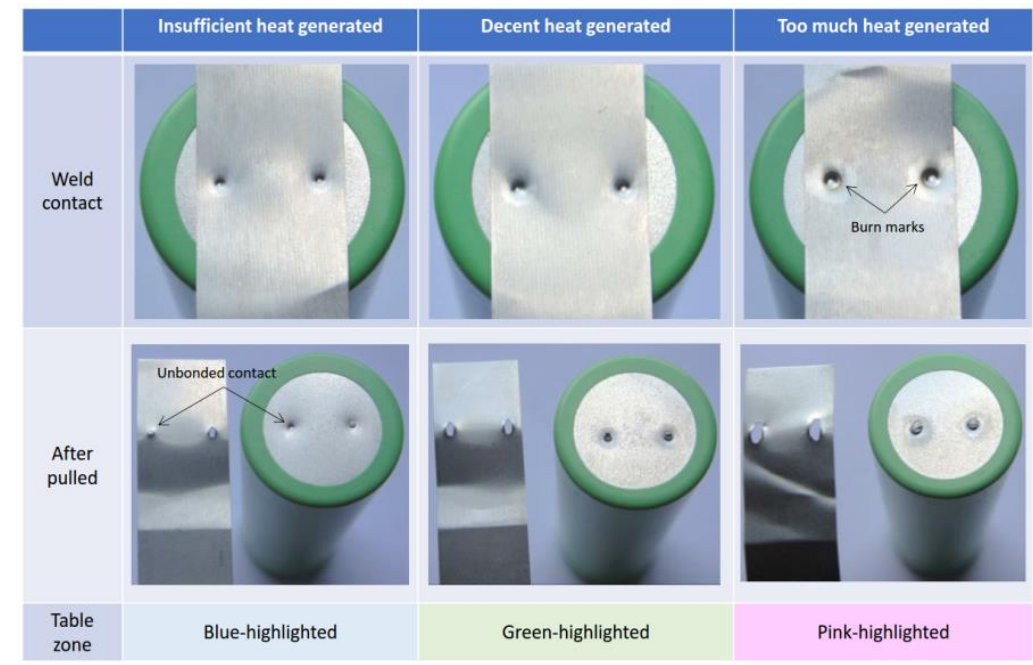

Fig. 28. Results of weld contacts with different amount of heat generated. 


\section{Conclusion}

This work was set out to study the effects of parameters that have influences on spot welding results, and implement the obtained information to conduct spot welding between Hilumin $\mathbb{R} /$ nickel connecting tab and Samsung INR18650-15L Li-ion battery cells using Miyachi FD120 series weld head. For the electrode tip geometry, the round electrode tip is better than the other electrode tip geometries in term of the life time and the lower oxidation. For the distance between welding electrode parameter, it does not have influences on electric current flow invisibility, but it has more deformation at the negative side in case of using the short distance. In practice, if the short distance is required in order to weld both positive and negative sides without changing the distance, the position of the welding spots should be located in the regions far away from the center of the battery cell. For the connecting tab design, having a slot on the connecting tab can make more electric current flow through the spot welding area. For the connecting tab material, the temperature and the electric current using nickel tab is higher than using Hilumin ${ }^{\circledR}$ at the same stage of maximum supply voltage. Therefore, nickel uses lower power consumption than Hilumin ${ }^{\circledR}$. For the welding force setup, inadequate welding force can lead to a weak connection between welding surfaces causing metal splash and results in poor weld result. In contrast, too much welding force applied to the welding contact causes excessive deformation of work-pieces creating a large touching welding surface rather than a small one. This produces dispersed current flow paths and consequently, insufficient heat is generated which results in poor strength of weld contacts. For the welding time setup, insufficient amount of heat is produced with too short welding time setting, results in weak or unboned weld contacts. Too long welding time can lead to excessive amount of heat generated forming burn mark in the middle region between two weld spots, which can further develop into rust under humid environment. For the maximum supply voltage setup, using too high maximum supply voltage setting could allow redundant heat generated causing excessive deformation of work-pieces, and also burn mark around the weld spots as well as the middle region between them. After having the results obtained from simulations and experiments, the results for proper parameter range for spot welding on positive and negative terminals can be found in the green-highlighted zone of Table 1 and Table 2 respectively. Although the obtained parameter setting can be used as a reference for welding metal connecting tabs on $18650 \mathrm{Li}$-ion battery cells to create battery packs, in practice, if material or geometry of connecting tab is changed, changes in parameter setting is likely to be necessary to achieve optimal welding results.

\section{References}

[1] M. J. Brand, P. A. Schmidt, M. F. Zaeh, and A. Jossen, "Welding techniques for battery cells and resulting electrical contact resistances," Journal of Energy Storage, vol. 1, no. 1, pp. 7-14, 2015.

[2] Miyachi Europe GmbH, General References on Resistance Welding. 2013.

[3] Tata Steel Europe Limited, Hilumin® Ultra-Clean Nickel-Plated Steel. 2013.

[4] Miyachi Europe GmbH, "Betriebsanleitung mit Konformitätserklärung FD120 - F120," 2014.

[5] T. J. SNOW CO. MIB-200A AC Inverter Welding Power Supply [Online]. Available: http://www.tjsnow.com/supplies/miyachi/MIB-200A.htm [Accessed: 5 May 2015].

[6] dealbay24. (2015). Nickel Lötbrücken [Online]. Available: http://www.dealbay24.de [Accessed: 15 May 2015].

[7] rcGroups. (2015). How To Build a Battery Pack from A123 Cells [Online]. Available: http://www.rcgroups.com [Accessed: 15 May 2015].

[8] WikimediaCommons. Fill:H-Verbinder Y-Ableiter Streifenverbinder-Ableiter [Online]. Available: http://commons.wikimedia.org/wiki/File:HVerbinder_Y-Ableiter_Streifenverbinder-Ableiter.jpg. [Accessed: 15 May 2015]

[9] EngineeringToolBox.MetalsMeltingTemperatures [Online]. Available: http://www.engineeringtoolbox.com. [Accessed: 15 May 2015]. 